\title{
Óxido Nítrico, Doenças Cardiovasculares e Exercício Físico
}

\author{
Nitric Oxide, Cardiovascular Disease and Physical Exercise \\ Anderson Saranz Zago e Angelina Zanesco \\ Universidade Estadual Paulista - Rio Claro, SP
}

A hipertensão arterial (HA) possui alta incidência na população mundial e sua etiologia é multifatorial, envolvendo fatores genéticos, ambientais e psicológicos ${ }^{1,2}$. Sua prevalência tem aumentado de maneira crescente, sobretudo entre mulheres, negros e idosos. Mais de $50 \%$ dos indivíduos entre 60 e 69 anos e aproximadamente $3 / 4$ da população acima dos 70 anos são afetados pela $\mathrm{HA}^{1}$. Além disso, existe uma correlação positiva e direta entre os níveis de pressão arterial elevada e o risco de doenças cardiovasculares, independentemente do sexo, faixa etária e etnia ${ }^{1,2}$. Assim, a compreensão dos mecanismos celulares e moleculares envolvidos na gênese da HA é fundamental para a realização de medidas preventivas e/ou terapêuticas com o objetivo de controlar os níveis pressóricos e, conseqüentemente, reduzir os riscos cardiovasculares associados à HA. Dessa forma, esta revisão busca abordar os mecanismos envolvidos no controle da pressão arterial, enfocando o papel do endotélio vascular e a importância do exercício físico na regulação da produção de óxido nítrico pelas células endoteliais.

\section{Regulação da pressão arterial}

A pressão arterial (PA) pode ser controlada essencialmente por dois mecanismos: regulação neural, que é feita primariamente pelo sistema nervoso autônomo (associado aos barorreceptores e quimiorreceptores) e a regulação humoral, que é feita por uma variedade de substâncias liberadas por diferentes tipos celulares, como as células endoteliais e as células justaglomerulares. Alterações em um ou ambos os mecanismos de controle da PA (neural e/ou humoral), poderão resultar em elevação dos níveis pressóricos, instalando-se assim um quadro de $\mathrm{HA}^{3}$.

O sistema nervoso autônomo é composto pelo sistema nervoso simpático e pelo parassimpático que têm papel fundamental no controle da PA, sendo ambos muito estudados no sentido de compreender a sua participação na gênese e/ ou manutenção do estado hipertensivo. Os barorreceptores, mediadores primários do sistema nervoso autônomo no controle da PA e da freqüência cardíaca, são terminações nervosas que respondem à deformação ou estiramento das paredes dos vasos onde estão localizados. Os seios carotídeos e a croça da aorta são os locais de maior concentração dessas aferências e com maior expressão fisiológica ${ }^{3}$. Os seios carotídeos são dilatações das artérias carótidas internas que possuem a parede dessa região mais fina e com maior quantidade de tecido elástico do que de tecido muscular liso comparada a outras porções da parede arterial. A inervação sensorial dessa área é provida pelos ramos do nervo glossofaríngeo, enquanto que a inervação dos barorreceptores aórticos é feita pelo nervo aórtico ${ }^{4}$. As aferências barorreceptoras terminam no núcleo do trato solitário (NTS), onde normalmente são efetuadas as respostas de inibição da descarga simpática e intensificação da resposta vagal. O aumento da PA promove estimulação dos barorreceptores aórticos e carotídeos, desencadeando reflexamente uma inibição da descarga simpática, enquanto que uma queda da PA produz o efeito contrário ${ }^{3,5}$. Os quimiorreceptores estão localizados na aorta e carótida e respondem a estímulos químicos de pressão parcial de oxigênio $\left(\mathrm{O}_{2}\right)$, pressão parcial de dióxido de carbono $\left(\mathrm{CO}_{2}\right)$ e $\mathrm{pH}$, sendo extremamente importantes nos estados de anoxia e/ou hipóxia ${ }^{3,6-8}$.

O controle humoral é feito por diversos tipos celulares. As células justaglomerulares presentes nos rins liberam a renina que na corrente sangüínea vai atuar sobre uma proteína plasmática, chamada angiotensinogênio, formando a angiotensina I. A angiotensina I, por sua vez, é convertida em angiotensina II, sendo que essa conversão ocorre quase que totalmente nos pequenos vasos dos pulmões, catalisada pela enzima conversora presente no endotélio dos vasos pulmonares. A angiotensina II é um potente vasoconstritor e sua ligação com os receptores $\mathrm{AT}_{1}$, presentes na musculatura lisa vascular, ativa a proteína $G$ com conseqüente ativação de fosfolipase C- $\beta$ e formação de 1,4,5-trifosfato e diacilglicerol que induz o aumento da concentração intracelular de cálcio e ativação da proteína $C$ quinase, promovendo vasoconstrição e, conseqüentemente, elevação da $\mathrm{PA}^{9}$. Cabe salientar que a resposta à angiotensina II é bifásica na maior parte dos leitos vasculares, pois ocorre uma discreta vasodilatação desencadeada pela ativação dos receptores $\mathrm{AT}_{2}$ presentes nas células endoteliais, seguida de potente vasoconstrição pela ativação dos receptores $\mathrm{AT}_{1}$ presentes nas células vasculares lisas. A angiotensina II promove também hipertrofia ventricular esquerda, vasoconstrição renal, contração de células mesangiais, aumento da atividade da bomba de $\mathrm{Na}^{+} /$ $\mathrm{H}^{+}$proximal e distal dos túbulos renais, inibição da secreção de renina e liberação de aldosterona ${ }^{3,9}$.

As células endoteliais desempenham relevante papel no controle do tônus cardiovascular, regulando a vasomotricidade,

\section{Palavras-chave}

Óxido nítrico, doenças cardiovasculares, exercício físico. 


\section{Atualização Clínica}

a permeabilidade vascular, o metabolismo de substâncias endógenas e exógenas e a atividade plaquetária e leucocitária ${ }^{10}$.

Furchgott e Zawasdski ${ }^{11}$ foram os primeiros pesquisadores a demonstrar a importância do endotélio no controle do tonus vascular. Os autores relataram que a vasodilatação induzida pela acetilcolina era dependente da presença de um endotélio íntegro, e que as células endoteliais liberavam um fator de relaxamento, denominado fator de relaxamento derivado do endotélio (EDRF). Além da acetilcolina, verificouse posteriormente que outros agonistas como a histamina, a bradicinina, o ATP, a trombina, a noradrenalina, a angiotensina e a serotonina também eram capazes de liberar EDRF. Estudos mostram que as células endoteliais são capazes de sintetizar várias substâncias vasoativas, que foram classificadas em fatores relaxantes e fatores contráteis. Os fatores relaxantes derivados do endotélio são: o óxido nítrico $(\mathrm{NO})$, a prostaciclina $\left(\mathrm{PGI}_{2}\right)$ e o fator hiperpolarizante derivado do endotélio (EDHF) ${ }^{11-14 .}$ Os fatores contráteis derivados do endotélio são a endotelina e o tromboxano ${ }^{15}$.

Em especial, o NO produzido pelas células endoteliais desempenha um papel de grande importância no controle cardiovascular, tanto no controle da resistência periférica vascular como na agregação plaquetária ${ }^{14}$. O NO é um potente vasodilatador e assim seu papel no controle da PA é extremamente relevante. Além disso, o NO inibe a agregação plaquetária impedindo a formação de trombos e, conseqüentemente, prevenindo os processos de tromboses e doenças atero-trombóticas ${ }^{14,16}$.

\section{Síntese do óxido nítrico}

A biossíntese do NO compreende uma das funções mais importantes do metabolismo da L-arginina no organismo. O NO é formado a partir do nitrogênio da guanidina presente na L-arginina, sob a ação catalítica da enzima sintase do óxido nítrico, gerando concentrações equimolares de L-citrulina ${ }^{16-18}$. O processo de formação do NO está ilustrado na figura 1.

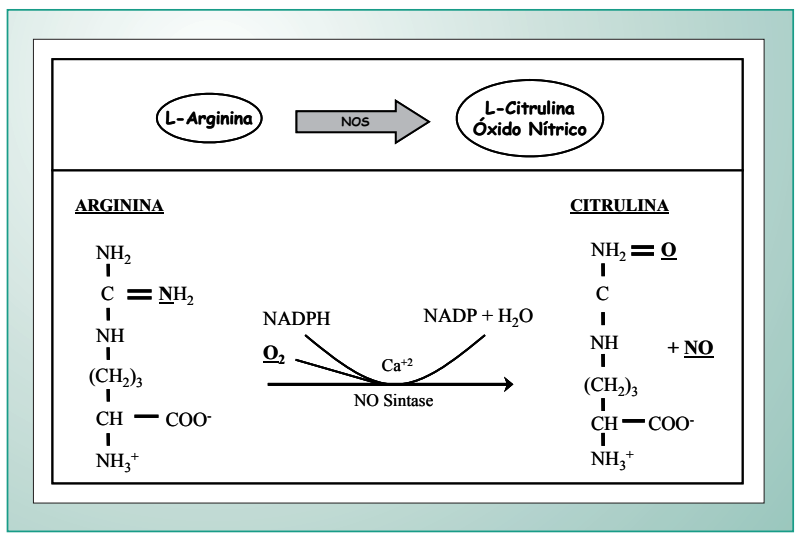

Fig. 1 - Esquema ilustrativo da formação do óxido nítrico a partir o metabolismo da Arginina pela ativação da enzima Óxido Nítrico Sintase (adaptado de LEHNINGER, 2002).

Para que a síntese do NO se realize é necessário que uma enzima seja ativada, desencadeando todo o processo de sua formação. Assim, a síntese de NO ocorre somente a partir da ativação da síntese do óxido nítrico (NOS) que existe em duas isoformas: a isoforma constitutiva e a induzível ${ }^{18-20}$.

As isoformas constitutivas (cNOS) são originalmente encontradas no endotélio e nos neurônios, sendo então denominadas de eNOS (NOS endotelial) e nNOS (NOS neuronal), respectivamente. $\mathrm{A}$ isoforma $\mathrm{nNOS}$, comumente chamada de isoforma I ou de bNOS (brain NOS), é encontrada no cérebro, na medula espinhal, nos gânglios simpáticos, em glândulas adrenais, nos neurônios nitrérgicos e em outras estruturas como células epiteliais de pulmões, útero e estômago, células da mácula densa do rim, células da ilhota pancreática e do músculo esquelético ${ }^{16}$. A isoforma eNOS, também chamada de isoforma III, está ligada à membrana das células endoteliais, regulando o tônus da célula muscular lisa vascular, bem como a adesão e agregação plaquetária. A eNOS também pode ser encontrada em sinciciotrofoblastos, células epiteliais tubulares do rim, células intersticiais do cólon e hipocampo. Ambas as isoformas encontram-se presentes nas células e são estimuladas por uma cascata bioquímica que pode ser dependente ou independente de íons cálcio $\left(\mathrm{Ca}^{2+}\right)$. Assim, a ativação da NOS constitutiva é dependente da elevação de íons $\mathrm{Ca}^{2+}$ nas células endoteliais, como será visto posteriormente com mais detalhes. Tanto a eNOS quanto a nNOS requerem um doador de elétron, a nicotinamida adenina dinucleotídeo fosfato reduzida (NADPH), e cofatores como a flavina adenina dinucleotídeo (FAD), a flavina mononucleotídeo (FMN) e a tetrahidrobiopterina $\left(\mathrm{BH}_{4}\right)^{14,21,22}$. Em humanos e, presumivelmente, na maioria das outras espécies, essas isoformas são codificadas por três diferentes genes localizados em três cromossomos distintos ${ }^{22}$.

A isoforma induzível (iNOS) é ativada a partir de alguns estímulos patológicos, como, por exemplo, lipopolissacarídeos bacterianos (LPS), citocinas, incluindo a interleucina-1, endotoxinas e fator de necrose tumoral, e são independentes de ions $\mathrm{Ca}^{2+}$. Essa isoforma pode ser expressa em uma grande variedade de tipos celulares incluindo macrófagos, linfócitos, neutrófilos, eosinófilos, células de Kupffer, hepatócitos e células epiteliais. Uma das grandes diferenças entre a NOS constitutiva e a induzível é que a iNOS é capaz de liberar grandes quantidades de NO por períodos de tempo relativamente longos, podendo gerar alguns efeitos exagerados, produzindo respostas tóxicas ao organismo, enquanto que a cNOS produz pequenas quantidades de $\mathrm{NO}$ e por tempo menor $^{22-25}$. Apesar de existirem diferenças entre as isoformas de NOS, todas elas atuam no sentido de catalisar a oxidação do átomo de nitrogênio terminal do grupamento guanidino da L-arginina, formando quantidades equimolares de $\mathrm{NO}$ e L-citrulina ${ }^{18-20}$, conforme observado na figura 1.

A ativação da NOS e a conseqüente síntese de NO pelas células endoteliais ocorre a partir de estímulos que podem ser químicos ou físicos. Os estímulos químicos são originados da interação de agonistas endógenos/exógenos com receptores específicos presentes nas células endoteliais, como, por exemplo, a acetilcolina, o ATP, e a bradicinina. A interação agonista-receptor, na célula endotelial, promove a formação de inositol trifosfato (IP3) que, por sua vez, induz a liberação de íons $\mathrm{Ca}^{2+}$ do retículo endoplasmático, eleva os níveis de $\mathrm{Ca}^{2+}$ intracelular, formando o complexo cálcio-camodulina, 
ativando a enzima NOS que irá atuar na L-arginina, gerando a formação do NO pelo endotélio ${ }^{22,26}$

O estímulo físico é feito pela força que o sangue exerce sobre a parede das artérias, denominada força de cisalhamento, ou shear stress. O mecanismo pelo qual o shear stress promove a formação de $\mathrm{NO}$ ainda não está completamente esclarecido. Sabe-se que as células endoteliais possuem mecanorreceptores, que podem ativar diretamente as proteínas G, os canais iônicos e as enzimas do grupo das proteínas quinases e fosfatases que vão promover a formação de segundos mensageiros, desencadeando uma série de reações químicas, que envolvem a participação dos íons cálcio, até a vasodilatação propriamente dita ${ }^{27-30}$.

Assim, as células endoteliais comportam-se de forma dinâmica e respondem às alterações tanto dos estímulos físicos (shear stress) quanto aos estímulos químicos, promovendo a síntese e liberação de substâncias vasoativas ${ }^{30}$.

Uma vez liberado, o NO difunde-se rapidamente da célula geradora para a célula-alvo ou, mais particularmente, das células endoteliais para a musculatura lisa do vaso sangüíneo. Na célula muscular lisa, o NO irá ativar uma enzima catalítica, a guanilato ciclase solúvel (GCs). Essa ativação é feita pelo acoplamento do NO com o grupamento heme desta enzima (sítio receptor), que por sua vez irá formar o monofosfato de guanosina cíclico (GMPc), a partir da quebra do trifosfato de guanosina (GTP). A formação do GMPc promove a ativação da bomba de cálcio dentro da célula muscular lisa, diminuindo as concentrações de cálcio intracelular que promoverá a redução do tonus vascular ${ }^{22}$. Outros mecanismos pelos quais a via NO/GMPc induz vasodilatação incluem inibição da geração de IP3 (na musculatura lisa), desfosforilação da cadeia leve de miosina, inibição do influxo de $\mathrm{Ca}^{2+}$, ativação de proteínas quinases, estimulação da $\mathrm{Ca}^{2+}$-ATPase de membrana e abertura de canais de $\mathrm{K}^{+29,31,32}$.

Assim, em vasos sangüíneos, o aumento intracelular de GMPc induz ao relaxamento do músculo liso vascular, e conseqüentemente, à vasodilatação. Em plaquetas, a formação de GMPc irá inibir a agregação plaquetária, fato que justifica a atuação do NO neste mecanismo, haja vista que o $\mathrm{NO}$ desencadeia a formação de $\mathrm{GMPc}^{26}$. No rim, isso desencadeará um aumento da excreção renal de sódio e a conseqüente perda de água e diminuição do volume sangüíneo ${ }^{33,34}$.

A figura 2 ilustra a síntese, liberação e ação do NO e o papel da eNOS na sua produção.

Conforme se pode observar na figura 2, os íons $\mathrm{Ca}^{2+}$ desempenham importante papel no controle do tônus vascular. Como a musculatura lisa não possui a troponina, proteína reguladora presente no músculo esquelético, que é ativada pelos íons $\mathrm{Ca}^{2+}$ para promover a contração muscular, a contração da musculatura lisa ocorre devido à combinação entre o cálcio e a calmodulina. Essa combinação ativa uma enzima fosforilativa, a miosina quinase, que tem a função de fosforilar as cadeias leves da miosina, adquirindo a capacidade de se fixar ao filamento de actina e realizar a contração muscular. Dessa forma, a diminuição da concentração de $\mathrm{Ca}^{2+}$ impediria a combinação cálcio/calmodulina, gerando um relaxamento da musculatura lisa vascular e a conseqüente vasodilatação ${ }^{35}$.

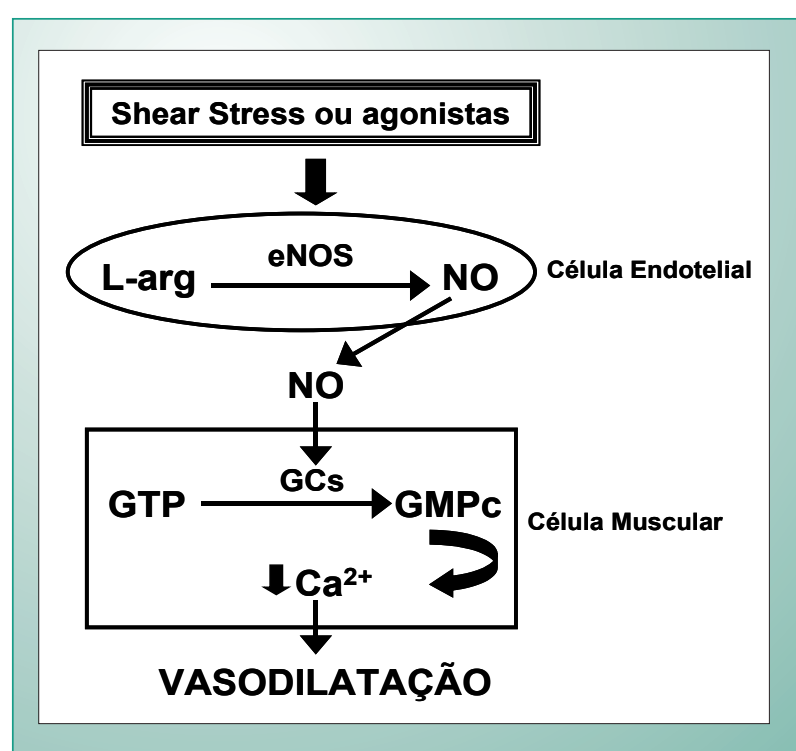

Fig. 2 - Esquema ilustrativo da síntese, liberação e ação do NO e o papel da eNOS na sua produção.

\section{Papel do NO nas doenças cardiovasculares}

O papel do NO no sistema cardiovascular foi extensamente estudado, sendo atribuído ao NO um papel protetor na HA, na aterosclerose, na doença arterial coronariana e nas doenças tromboembólicas ${ }^{32,34,36-40}$.

Diversas doenças, como as dislipidemias, a aterosclerose e a HA, apresentam em sua gênese e/ou em seus mecanismos, alterações na função endotelial. Assim, a disfunção endotelial, caracterizada por menor produção e/ou biodisponibilidade de $\mathrm{NO}$, é um dos fatores que contribuem para o aparecimento das doenças cardiovasculares ${ }^{40}$.

\section{Dislipidemias e aterosclerose}

As dislipidemias englobam a hipertrigliceridemia e a hipercolesterolemia. A hipertrigliceridemia é definida como níveis de triglicérides com valores acima de 200 mg/dL, enquanto que a hipercolesterolemia é definida como elevação da concentração plasmática de colesterol acima de $240 \mathrm{mg} / \mathrm{dL}^{2}$. Na hipercolesterolemia, a quantidade de lipoproteína de baixa densidade (LDL) é alta e sua elevada concentração na circulação favorece sua oxidação (LDL-ox). O processo de aterosclerose está estreitamente relacionado aos níveis plasmáticos de LDL colesterol, especialmente após a sua oxidação. Acredita-se que a presença de LDL-ox seja a primeira etapa no processo de desenvolvimento da aterosclerose $\mathrm{e}^{41}$, e sua oxidação está diretamente relacionada a sua concentração plasmática. Elevada concentração de LDL colesterol promove uma saturação do seu sistema de remoção, acarretando assim um aumento no seu tempo de permanência na circulação e, conseqüentemente, sua oxidação. A molécula de LDL pode ser oxidada por íons cálcio, lipoxigenases (produzidas pelas células endoteliais), mieloperoxidases (secretadas pelos fagócitos) e espécies reativas de oxigênio ${ }^{42}$, desencadeando todo o processo aterosclerótico. 


\section{Atualização Clínica}

O NO produzido pelas células endoteliais desempenha importante papel protetor na aterosclerose, inibindo a oxidação das moléculas de LDL colesterol e impedindo a agregação plaquetária ${ }^{10,21}$. O mecanismo pelo qual o NO impede a formação da molécula de LDL-ox, se dá por meio de sua ação antioxidante (que é dependente da concentração), impedindo a formação de ânions superóxidos (radicais livres), que promovem a oxidação da molécula de LDL colesterol. A ação antiagregante do NO é devida a sua ligação com a molécula de guanilatociclase, que induz a formação de guanilato monofosfato cíclico (GMPc), que promove a redução da concentração de íons cálcio dentro da plaqueta, inibindo sua ativação e agregação ${ }^{43,44}$.

A formação de LDL-ox promove eventos celulares de recrutamento de leucócitos para a região vascular afetada, os quais irão produzir substâncias deléterias para as células endoteliais (interleucinas), reduzindo a produção de $\mathrm{NO}$ e/ou sua biodisponibilidade. Estudos clínicos mostram que indivíduos com hipercolesterolemia apresentam diminuição na produção dos fatores relaxantes derivados do endotélio ${ }^{38}$. Por outro lado, outros estudos mostram que a produção do NO não é afetada pelos níveis elevados de colesterol circulantes, mas que a produção de espécies reativas de oxigênio como os ânions superóxidos (ONOO-) reagem com a molécula de NO produzida, diminuindo a sua disponibilidade para as células e favorecendo os processos tromboembólicos. Isso estaria acontecendo devido ao fato de a hipercolesterolemia estar associada ao aumento da atividade da NAD/NADPH oxidase, fatores fundamentais para a formação dos ânions superóxidos ${ }^{38,45,46}$.

A redução de NO promove a agregação plaquetária, a hiperplasia e a hipertrofia das células musculares lisas, acarretando significativa redução da luz dos vasos e, conseqüentemente, a isquemia dos tecidos. No coração, um fluxo sangüíneo reduzido nos vasos coronarianos acarreta deficiência no funcionamento do miocárdio ${ }^{47}$. Estudos em animais de laboratório mostraram que o comprometimento do processo relaxante dependente do endotélio em vasos coronarianos pode provocar insuficiência cardíaca crônica ${ }^{32,38,39,48}$. A melhora do perfil lipídico restaura a produção e/ou biodisponibilidade do NO. Dessa forma, a redução dos níveis plasmáticos de LDL colesterol e/ou o aumento da fração de HDL colesterol que faz o transporte reverso da molécula de LDL colesterol, são medidas extremamente benéficas para o organismo na prevenção e/ou tratamento da aterosclerose ${ }^{37}$. Uma das formas de se reduzir os níveis de LDL colesterol circulantes é através da prática regular de exercícios físicos. Diversos trabalhos mostram que indivíduos fisicamente ativos possuem um perfil lipídico dentro dos valores normais quando comparados com indivíduos sedentários ${ }^{38,40,49}$. Assim, a prática de exercício físico regular é uma importante medida de prevenção e/ou tratamento das dislipidemias, cujos mecanismos envolvem a restauração da função das células endoteliais e a melhora do perfil lipídico ${ }^{49}$.

\section{Hipertensão arterial}

A HA é uma doença de etiologia multifatorial que causa lesões em vários órgãos, tais como: coração, cérebro, vasos, rins e retina, sendo considerada um dos mais importantes fatores de risco para o desenvolvimento das complicações cardiovasculares como, por exemplo, $40 \%$ das mortes por acidentes vasculares encefálicos e $25 \%$ das doenças coronarianas. Para o diagnóstico da HA, além dos níveis tensionais, devem ser considerados os fatores de risco, a lesão de órgãos-alvos e as cormobidades associadas. A HA é basicamente uma doença assintomática, mas, por outro lado, pode às vezes apresentar alguns sintomas tais como cefaléia, geralmente matutina e de localização occipital, tonturas, escotoma, tinido, palpitações e desconforto precordial ${ }^{3}$.

O efeito do exercício físico regular sobre a HA tem sido estudado tanto em animais de laboratório como em seres humanos. Dados experimentais mostram que a redução da PA após exercício é maior em indivíduos hipertensos do que em normotensos. A redução dos valores de PA sistólica e diastólica decorrente da atividade física varia de 18-20 mmHg e de 7-9 $\mathrm{mmHg}$, respectivamente, em humanos com hipertensão leve ou moderada; e em indivíduos normotensos, a redução é de 8-10 mmHg e 3-5 mmHg, respectivamente ${ }^{50}$.

Os efeitos benéficos do exercício físico sobre o controle da PA têm sido abordados sob dois aspectos: preventivos e terapêuticos. Os aspectos preventivos envolvem a promoção da saúde e prevenção do estado hipertensivo em sujeitos com maior risco de desenvolver a doença. Os aspectos terapêuticos envolvem, além da redução dos níveis pressóricos e alteração do perfil lipídico, diminuição da mortalidade, mesmo quando não ocorre redução dos níveis pressóricos.

Estudos têm demonstrado que variações na intensidade do exercício têm diferentes efeitos na PA em ratos espontaneamente hipertensos (SHR) e em humanos ${ }^{51,52}$. Os exercícios aeróbios ou dinâmicos como ciclismo, natação, subir e descer escadas, corrida leve em esteira ou em movimento (trotar) são mais eficazes como terapia alternativa (ou concomitante com terapia farmacológica) no tratamento da hipertensão leve ou moderada ${ }^{53-57}$. Os programas de condicionamento físico para hipertensos preconizam que os exercícios devem ser realizados, no mínimo, três vezes por semana, durante pelo menos 30 minutos por sessão. Exercícios de baixa a moderada intensidade (40-70\% de $\mathrm{VO}_{2}$ máx) acarretam melhores resultados na redução da PA do que exercícios de maior intensidade ( $>75 \%$ de $\mathrm{VO}_{2}$ máx $)^{56}$. A maioria dos estudos mostra que a redução da PA em sujeitos com hipertensão essencial ocorre dentro de três semanas a três meses após o início do treinamento físico, e essa redução é mantida enquanto se mantém o treinamento ${ }^{40,56}$.

Vários estudos em animais mostram que a inibição da NOS por análogos da L-arginina, o L-NAME, por exemplo, reduz a produção de NO e gera HA de maneira dose-dependente er-63 $^{58}$. A administração diária de L-NAME (20 mg/rato/dia) a ratos leva à inibição crônica da síntese de NO, produzindo elevação de PA, que é acompanhada por alterações funcionais e estruturais do rim $^{61,64}$ e do coração ${ }^{65}$. No coração, as anormalidades cardíacas incluem principalmente hipertrofia ventricular e focos de necrose e fibrose ${ }^{65-68}$. As lesões cardíacas parecem ser decorrentes da intensa vasoconstrição coronariana provocada pelo L-NAME, que leva a uma menor oferta de oxigênio e nutrientes aos cardiomiócitos, provocando a morte dos mesmos. O miócito morto dá então origem a um tecido 
conjuntivo cicatricial do tipo fibrótico que pode trazer um comprometimento relativamente sério ao sistema cardíaco. Esses estudos reforçam o efeito benéfico do NO no sistema cardiovascular e, particularmente, no controle da PA.

\section{Óxido nítrico e atividade física}

Até a década de 90, diversos estudos mostravam a importância da prática de exercício físico regular na prevenção e tratamento de diversas doenças, em especial as doenças cardiovasculares. No entanto, o que ou quais fatores estariam envolvidos nos efeitos benéficos do exercício físico não eram conhecidos. A partir da descoberta da molécula de NO, inúmeros trabalhos foram realizados avaliando-se o efeito do exercício físico sobre as células endoteliais, sobre a produção de fatores relaxantes e sobre a sua correlação com os efeitos benéficos produzidos pelo exercício físico $27,28,40,69,70$.

Estudos em seres humanos e em animais de laboratório mostram que o shear stress induzido pelo exercício físico é um poderoso estímulo para a liberação de fatores vasorrelaxantes produzidos pelo endotélio vascular, como o $\mathrm{NO}$ e o fator hiperpolarizante derivado do endotélio (EDHF), acarretando assim redução dos valores de $\mathrm{PA}^{40,71}$. Ficou demonstrado que o treinamento físico moderado aumenta o relaxamento da musculatura lisa vascular e não vascular e que esse maior relaxamento seria devido à maior produção de EDHF e NO pelas células endoteliais em resposta ao exercício físico ${ }^{28,72-74}$. Além disso, observou-se que o shear stress induzido pelo exercício físico aumenta a expressão da NOS endotelial e neuronal $27,70,75$.

Assim, os efeitos benéficos da prática de exercício regular sobre as doenças cardiovasculares foram associados, principalmente, à maior produção de agentes vasodilatadores derivados do endotélio ( $\mathrm{NO}$ e EDHF), com conseqüente redução da resistência vascular periférica, diminuição dos níveis de LDL colesterol e inibição da agregação plaquetária ${ }^{40,76}$. Esse efeito estaria gerando uma melhora nos níveis pressóricos e, conseqüentemente, uma menor incidência de HA.

É importante salientar que o exercício físico parece ter efeito de proteção na integridade do endotélio, quer seja aumentando a produção de $\mathrm{NO}$ em vasos com endotélio íntegro, quer restaurando a disfunção endotelial. Higashi et a ${ }^{28}$ confirmam essa afirmação ao comparar a responsividade

\section{Referências}

1. Dórea EL, Lotufo PA. Epidemiologia da hipertensão arterial sistêmica. Hipertensão. 2004; 7: 86-9.

2. MacKay J, Mensah GA (eds) > The atlas of heart disease and stroke. Geneva: WHO; 2004.

3. Guyton AC, Hall JE. Textbook of medical physiology. 9ªed. Philadelphia: W.B. Saunders Company, 1996.

4. Milnor GL. Hypothalamic control of baroreceptor. Am J Physiol. 1970; 218: 124-31.

5. Krieger EM. Neurogenic hypertension in the rat. Circ Res. 1964; 15: 511-21. vascular entre indivíduos hipertensos e normotensos diante do exercício físico aeróbio. Os autores observaram que, voluntários normotensos apresentaram relaxamento dos vasos sangüíneos significativamente maior do que o grupo hipertenso após a sessão de exercício físico, sugerindo a existência de disfunção endotelial no grupo hipertenso. E, comparando grupos hipertensos de controle e praticantes de um programa de 12 semanas de atividade física aeróbia, a responsividade vascular foi aumentada no grupo que se exercitava, ou seja, a atividade física contribui para restabelecer a função endotelial. Dessa forma, o papel da atividade física torna-se essencial para o controle cardiovascular, tanto pelo benefício no controle da PA quanto como inibidor da agregação plaquetária e da formação de LDL-ox, atuando de forma preventiva e/ou terapêutica em diversas patologias como a aterosclerose, $\mathrm{HA}$ e as dislipidemias.

\section{Considerações finais}

O papel do NO nas doenças cardiovasculares tem merecido destaque em todas as áreas de conhecimento. A ação benéfica do NO na PA, nas doenças tromboembólicas e nas dislipidemias está bem estabelecida. A integridade do endotélio é fundamental para a produção do NO e o exercício físico tem sido empregado como uma ferramenta extremamente importante para manter a função endotelial e/ou restaurá-la. Assim, a prática regular de atividade física é fundamental para a manutenção da saúde e prevenção e/ou tratamento das doenças cardiovasculares, entre outras. O Brasil possui um índice elevado de indivíduos sedentários sendo necessárias políticas públicas voltadas para o investimento na área de esporte e lazer. Além disso, a qualificação profissional do educador físico, dentro da área de saúde, é de grande relevância como estratégia educacional na redução da prevalência de doenças cardiovasculares na população brasileira.

\section{Agradecimentos}

Fundação de Amparo à Pesquisa do Estado de São Paulo (FAPESP) - no do processo: 04/07779-4.

\section{Potencial Conflito de Interesses}

Declaro não haver conflitos de interesses pertinentes.
6. Machado BH, Mauad H, Glass ML. Transient changes in blood pressure during spontaneous deep breaths in rats with sinoaortic deafferentation. J Appl Physiol. 1992; 72: 920-4

7. Barros RC, Bonagamba LG, Okamoto-Canesin R, Oliveira M, Branco LG Machado BH. Cardiovascular responses to chemoreflex activation with potassium cyanide or hypoxic hypoxia in awake rats. Auton Neurosci. 2002; 97: 110-5.

8. Fernandes LG, Antunes VR, Bonagamba LG, Machado BH. Pressor response to chemoreflex activation in awake rats: role of vasopressin and adrenal medulla. Physiol Behav. 2005; 84: 39-44. 


\section{Atualização Clínica}

9. Cogolludo A, Vizcaíno FP, Tamargo J. New insights in the pharmacological therapy of arterial hypertension. Curr Opin Nephrol Hypertens. 2005; 14: 423-7.

10. Zanesco A, Antunes E. Células endoteliais. In: Carvalho HF, Buzato CBC Células: uma abordagem multidisciplinar, São Paulo: Manole, 2005.

11. Furchgott RF, Zawadzki JV. The obligatory role of endothelial cells in the relaxation of arterial smooth muscle by acetylcholine. Nature. 1980; 288: $373-6$

12. Ignarro LJ, Byrns R, Buga GM, Wood RS, Chaudhuri G. Pharmacological evidence that endothelium-derived relaxing factor is nitric oxide: use of pyrogallol and superoxide dismutase to study the endothelium-dependen and nitric oxide elicited vascular smooth muscle relaxation. J Pharmacol Exp Ther. $1987 ; 244: 1-9$

13. Palmer RMJ, Ferridge AG, Moncada S. Nitric oxide release accounts for the biological activity of endothelium-derived relaxing factor. Nature. 1987 327: 524-6.

14. Vanhoutte PM. Endothelial control of vasomotor function - From health to coronary disease. Circ J. 2003; 67: 572-5.

15. Yanagisawa M, Kurihara H, Kimura S, Goto K, Masaki T. A novel peptide vasoconstrictor, endothelin, is produced by vascular endothelium and modulates smooth muscle Ca2 + channels. J Hypertens. 1989; 6 (4 Suppl.): S188-91.

16. Moncada S, Palmer RM, Higgs EA. Nitric oxide: physiology pathophysiology and pharmacology. Pharmacol Rev. 1991; 43: 109-42.

17. Cohen RA, Vanhoutte PM. Endothelium-Dependent Hyperpolarization - Beyond Nitric Oxide and Cyclic GMP. Circulation. 1995; 92: 3337-49.

18. Lehninger A. Princípios da bioquímica. 3ạ ed. São Paulo: Sarvier; 2002.

19. Palmer RMJ, Ashton DS, Moncada S. Vascular endotelial cells synthesize nitric oxide from L-arginine. Nature. 1988; 333: 664-6.

20. Bredt DS, Hwang PM, Glatt CE, Lowenstein C, Reed RR, Snyder SH. Cloned and expression nitric oxide synthase structurally resembles cytocrome P-450 reductase. Nature. 1991; 351: 714-8.

21. Mombouli JV, Vanhoutte PM. Endothelial dysfunction: from physiology to therapy. J Mol Cell Cardiol. 1999; 31: 61-74.

22. Moncada S. Nitric Oxide in the vasculature: physiology and pathophysiology. An New York Acad Sci. 1997; 811: 60-7.

23. Schulz R, Triggle CR. Role of NO in vascular smooth muscle and cardiac muscle function. Trends Pharmacol Sci. 1994; 15: 255-9.

24. Dominiczack AF, Bohr DF. Nitric oxide and its putative role in hypertension Hypertension. 1995; 25: 1202-11.

25. Tibiriça E. Fisiopatologia em medicina cardiovascular. Rio de Janeiro: Revinter; 2001.

26. Moncada S. Physiological effects of nitric oxide. J Hypertens. 1994; 12: 35-9.

27. Sessa WC, Pritchard K, Seyedi N, Wang J, Hintze TH. Chronic exercise in dog increase coronary vascular nitric oxide production and endothelial cell nitric oxide synthase gene expression. Circ Res. 1994; 74: 349-53.

28. Higashi Y, Sasaki S, Sasaki N, Nakagawa K, Ueda T, Yoshimizu A, et al. Daily aerobic exercise improves reactive hyperemia in patients with essentia hypertension. Hypertension. 1999; 33:591-7.

29. Fisher AB, Chien S, Barakat Al, Nerem RN. Endothelial cellular response to altered shear stress. Am J Physiol. 2001; 281: L529-L533.

30. Boo YC, Jo H. Flow-dependent regulation of endothelial nitric oxide synthase: role of protein kinases. Am J Physiol. 2005; 285: C499-C508.

31. Murad F, Forstermann U, Nakane M, Schimidt H, Pollock J, Sheng H, et al. The nitric oxide-cyclic GMP signal transduction pathway in vascular smooth muscle preparations and other tissues. Jpn J Pharmacol. 1992; 58: 150-7.

32. Mcardle WD, Katch FI, Katch VL. Fisiologia do exercício. $5^{\underline{a}}$ ed. Rio de Janeiro: Guanabara Koogan; 2003.

33. Murad F, Forstermann U, Nakane M, Pollock J, Tracey R, Matsumoto T, et al. The nitric oxide-cyclic GMP signal transduction system for intracellular and intercellular communication. Adv Second Messenger Phosphoprotein Res.
1993; 28: 101-9.

34. Scott-Burden T, Vanhoutte PM. The endothelium as a regulator of vascular smooth muscle proliferation. Circulation. 1993; 87 (Suppl. 5): 51-5.

35. Webb RC. Smooth muscle contraction and relaxation. Adv Physiol Educ. 2003; 27: 201-6.

36. Rosselli M, Imthurn B, Keller PJ, Jackson EK, Dubey RK. Circulating Nitric Oxide (nitrite/nitrate) levels in postmenopausal women substituted with $17 \beta$-estradiol and norethisterone acetate - A two-year follow-up study. Hypertension. 1995; 25: 848-53.

37. Best PJM, Berger PB, Miller VM, Lerman A. The effect of estrogen replacement therapy on plasma nitric oxide and endothelin-1 levels in postmenopausal women. Ann Int Med. 1998; 128: 285-8.

38. Drexler H, Hornig B. Endothelial dysfunction in human disease. J Mol Cell Cardiol. 1999; 31: 51-60.

39. Viaro F, Nobre F, Evora RB. Expressão das óxido nítrico sintase na fisiopatologia das doenças cardiovasculares. Arq Bras Cardiol. 2000; 74: 365-79.

40. Kingwell BA. Nitric oxide-mediated metabolic regulation during exercise: effects of training in health and cardiovascular disease. FASEB J. 2000; 14: 1685-96.

41. Parthasarathy S, Steinberg D, Witztum JL. The role of oxidized lowdensity lipoproteins in the patogénesis of atherosclerosis. Ann Rev Med. 1992; 43: 219-25.

42. Curi R, Pompéia C, Miyasaka CK, Procopio J. Entendendo a gordura - os ácidos graxos. São Paulo: Manole; 2002.

43. Moro MA, Russel RJ, Cellek S, Lizasoain I, Su Y, Darley-Usmar VM, et al. cGMP mediates the vascular and platelet actions of nitric oxide: confirmation using an inhibitor of the soluble guanylyl cyclase. Proc Natl Acad Sci. 1996; 93: $1480-5$

44. Hobbs AJ, Moncada S. Antiplatelet properties of a novel, non-NO-based soluble guanylate cyclase activator. Vasc Pharmacol. 2003; 40: 149-54.

45. Hettzer T, Ylä-Herttuala S, Luoma L, Kurz S, Münzel T, Just J, et al. Cigarette smoking potentials endothelial dysfunction of forearm resistance vessel in patients with hypercholesterolemia. Circulation. 1996; 93: 1346-53.

46. Rosenson RS. Statin in atherosclerosis: lipid-lowering agents with antioxidant capabilities. Atherosclerosis. 2004; 173: 1-12.

47. Bolad I, Delafontaine P. Endothelial dysfunction: its role in hypertension coronary disease. Curr Opin Cardiol. 2005; 20: 270-4.

48. Levine B, Kalman J, Mayer L, Fillit HM, Packer M. Elevated circulating levels of tumor necrosis factor in severe chronic heart failure. N Engl J Med. 1990; 323: 236-41.

49. Nunes APOB, Rios ACS, Cunha GA, Barretto ACP, Negrão CE. Efeitos de um programa de exercício físico não supervisionado e acompanhado a distância, via Internet, sobre a pressão arterial e composição corporal em indivíduos normotensos e pré-hipertensos. Arq Bras Cardiol. 2006; 86: 289-96.

50. Kenney MJ, Seals DR. Postexercise hypotension. Hypertension. 1993; 22: 653-64.

51. Tipton CM, Mathees RD, Bedford TG. Influence of training on the blood pressure changes during lower body negative pressure in rats. Med Sci Sports Exerc. 1982; 14: 81-90.

52. Grassi G, Seravalle G, Calboun DA, Mancia G. Physical training and baroreceptor control of sympathetic nerve activity in human. Hypertension. 1994; 23: 294-301.

53. Franklin BA, Seymour G, Timmis GC. Exercise prescription for hypertensive patients. Ann Med. 1991; 23: 279-87.

54. Negrão CE, Irigoyen MC, Moreira ED, Brum PC, Freire PM, Krieger EM. Effect of exercise training on RSNA, baroreflex control, and blood pressure responsiveness. Am J Physiol. 1993; 265: 365-70.

55. Gava NS, Veras-Silva AS, Negrão CE, Krieger EM. Low-intensity exercise training attenuates cardiac beta-adrenergic tone during exercise in spontaneously hypertensive rats. Hypertension. 1995; 26: 1129-33.

56. Pescatello LS, Franklin BA, Fagard R, Farguhar WB, Kelley GA, Ray CA, et al. American College of Sports Medicine position stand Exercise and 
Hypertension. Med Sci Sports Exerc. 2004; 36: 533-53.

57. Monteiro MF, Sobral Fo DC. Exercício físico e o controle da pressão arterial. Rev Bras Med Esporte. 2004; 10: 513-6.

58. Gardiner SM, Compton AM, Kemp PA, Bennett T. Regional and haemodynamic effects of NG-nitro-L-arginine methyl ester in conscious long evans rats. Br J Pharmacol. 1990; 101: 625-31.

59. Klabunde RE, Ritger RC, Helgren MC. Cardiovascular actions of inhibitors of endothelium-derived relaxing factors (nitric oxide) formation release in anesthetized dogs. Eur J Pharmacol. 1991; 199: 51-9.

60. Richard V, Berdeaux A, La Rochelle CD, Giudicelli JF. Regional coronary hemodynamic effects of two inhibitor of nitric oxide synthase in anesthetized, open-chest dogs. Br J Pharmacol. 1991; 104: 59-64.

61. Ribeiro MO, Antunes E, De Nucci G, Lovisolo SM, Zatz R. Chronic inhibition of nitric oxide synthase - A new model of arterial hypertension. Hypertension. 1992; 20: 298-303.

62. Zatz R, Bayliss C. Chronic nitric oxide inhibition model six years on. Hypertension. 1998; 32: 958-64.

63. Riado SR, Zanesco A, Barker LA, De Lucca IMS, Antunes E, De Nucci G. Long-term nitric oxide inhibition and chronotrotic response in rat isolated right atria. Hypertension. 1999; 34: 802-7.

64. Baylis C, Mitruka B, Deng A. Chronic blockade of nitric oxide synthesis in the rat produces systemic hypertension and glomerular damage. J Clin Invest. 1992; 90: 278-81.

65. Moreno Jr H, Metze K, Bento AC, Antunes E, Zatz R, De Nucci G. Chronic nitric oxide inhibition as a model of hypertensive heart muscle disease. Basic Res Cardiovasc. 1996; 91: 248-55.

66. Batal P, Pechanova O, Bernatova I, Stvrtina S. Chronic inhibition of NO synthesis produces myocardial fibrosis and arterial media hyperplasia. Histol Histopathol. 1997; 12: 623-9.

67. Devli AM, Brosnan MJ, Graham D, Morton JJ, Mcphaden AR, Mcintyre $M$, et al. Vascular smooth muscle cell polyploidy and cardiomyocyte hypertrophy due to chronic NOS inhibition in vivo. Am J Physiol. 1998; 274: H52-H59.

68. Luvara G, Pueyo ME, Phillippe M, Mandet C, Savoie F, Henrion D, et al Chronic blockade of $\mathrm{NO}$ synthase activity induces a proinflammatory phenotype in the arterial wall: prevention by angiotension II antagonism. Arterioscler Thromb Vasc Biol. 1998; 18: 1408-16.

69. DelpMD, Mcallister RM, Laughlin MH. Exercise training alters endotheliumdependent vasoreactivity of rat abdominal aorta. J Appl Physiol. 1993; 75: 1354-63.

70. Roberts K, Barnard RJ, Jasman A, Balon TW. Acute exercise increase nitric oxide synthase activity in skeletal muscle. Am J Physiol. 1999; 277: E390-E394.

71. Kuru O, Sentürk UK, Demir N, Yesilkaya A, Ergüler G, Erkiliç M. Effect of exercise on blood pressure in rats with chronic NOS inhibition. Eur J Appl Physiol. 2002; 87: 134-40

72. Griffin KL, Laughlin MH, Parker JL. Exercise training improves endotheliummediated vasorelaxarion after chronic coronary occlusion. J Appl Physiol. 1999; 87: 1948-56.

73. Clarkson P, Montgomery H, Mullen M, Donald A, Powe A, Bull T, et al. Exercise training enhances endothelial function in young men. J Am Coll Cardiol. 1999; 33: 1379-85

74. Claudino MA, Priviero FBM, Teixeira C, De Nucci G, Antunes G, Zanesco A. Improvement in relaxation response in corpus cavernosun from trained rats. Urology. 2004; 63: 1004-8.

75. Shen W, Rundborg M, Wang J, Stewart JM, Xu X, Ochoa M, et al. Role of EDRF in the regulation of regional blood flow and vascular resistance at rest and during exercise in conscious dog. J Appl Physiol. 1994; 77: 165-72.

76. Kingwell B, Sherrard B, Jennings G, Dart A. Four weeks of cycle training increases basal production of nitric oxide from the forearm. Am J Physiol. 1997; 272: H1070-H1077. 